\title{
SOME NEW EXAMPLES OF NON-KÄHLER RICCI SOLITONS
}

\author{
Andrew S. DAnCer And McKenzie Y. WAng
}

AbSTRACT. We produce non-Kähler complete steady gradient Ricci solitons generalising those constructed by Bryant and Ivey.

Mathematics Subject Classification (2000): 53C25, 53C44

\section{Introduction}

In this article we continue our investigation of reductions of the Ricci soliton equations to ordinary differential equations. Recall that a Ricci soliton consists of a complete Riemannian metric $g$ and a complete vector field $X$ on a manifold satisfying the equation:

$$
\operatorname{Ric}(g)+\frac{1}{2} \mathrm{~L}_{X} g+\frac{\epsilon}{2} g=0
$$

where $\epsilon$ is a real constant and $L$ denotes the Lie derivative.

This equation is a generalisation of the Einstein equation, and it is natural to look for solutions by methods that have been fruitful in the Einstein case. In [DW] we set up the formalism for cohomogeneity one Ricci solitons and wrote down the resulting ODE system. We found families of explicit Kähler solutions generalising those of [Ko], [Ca], [ChV], [G], [PTV], [FIK], [PS] and [ACGT].

Most of the known examples of Ricci solitons are indeed Kähler. The exceptions of which we are aware are the homogeneous solitons on nilpotent Lie groups [La], the rotationally symmetric Bryant solitons [Bry] on $\mathbb{R}^{n}(n>2)$, Ivey's generalization of these solutions $[\mathrm{Iv}]$, as well as the expanding counterparts described in [Cetc] and [GK]. (Note that if $n=2$ the Bryant soliton is Hamilton's famous cigar soliton [Ha1], which is Kähler.) The Bryant solutions are warped products on a single factor, while those of Ivey involve two factors.

In this paper we shall focus on steady gradient Ricci solitons and generalise the Bryant-Ivey examples to produce complete steady solitons on warped products over an arbitrary number of positive Einstein factors (see Theorem 4.17). An important tool in our analysis is the observation that the general cohomogeneity one steady soliton equations always admit a Lyapunov function. This generalises the Lyapunov function in the Bryant-Ivey systems.

Recall that Hamilton has proved that steady gradient Ricci solitons occur as type II singularity models for the Ricci flow when the curvature operator is non-negative and the Ricci is positive ([Ha2] or Theorem 4.3.6 in [CaZ]). Our new steady solitons have non-negative Ricci curvature (Theorem 4.18) and always some negative sectional

Received by the editors May 13, 2008.

The second author is partially supported by NSERC Grant No. OPG0009421. 
curvatures. They have asymptotically paraboloid geometry (Theorem 3.10) and hence zero asymptotic volume ratio. The asymptotic scalar curvature ratio is infinite (cf Remark 4.19). Whether these steady solitons can be realised as blow-up limits of non-trivial Ricci flows seems to be an interesting question.

\section{Lyapunov functions}

We recall the set-up from [DW]. We consider a manifold $M$ with an open dense set foliated by diffeomorphic hypersurfaces $P_{t}$ of real dimension $n$. Assume the metric can be written as $g=d t^{2}+g_{t}$ where $g_{t}$ is a metric on $P_{t}$. We can view $t$ as arclength along a geodesic orthogonal to the hypersurfaces. Let $r_{t}$ denote the Ricci tensor of $g_{t}$, viewed as an endomorphism via $g_{t}$, and let $L_{t}$ denote the shape operator of the hypersurfaces (so $\dot{g}_{t}=2 g_{t} L_{t}$ ). Assume that the scalar curvature $R_{t}=\operatorname{tr}\left(r_{t}\right)$ and the mean curvature $\operatorname{tr}\left(L_{t}\right)$ are constant on each hypersurface. Furthermore, assume that the codifferentials $\delta^{\nabla^{t}} L_{t}$ vanish, where $L_{t}$ is viewed as a $T P_{t}$-valued 1-form on $P_{t}$.

The above assumptions are satisfied, for example, if $M$ is of cohomogeneity one with respect to an isometric group action, with no repeated summands in the isotropy representation of the principal orbits $P_{t}$. They are satisfied also when $M$ is a multiple warped product over an interval, which will be the setting of this paper.

We consider solitons of gradient type, i.e., where $X=\operatorname{grad} u$ for a function $u$. Equation (0.1) then becomes

$$
\operatorname{Ric}(g)+\operatorname{Hess}(u)+\frac{\epsilon}{2} g=0 .
$$

We will further suppose that $u$ is a function of $t$ only. In this setting, the above equation become the system ( $\mathrm{cf} \S 1$ of $[\mathrm{DW}]$ )

$$
\begin{aligned}
-\operatorname{tr}(\dot{L})-\operatorname{tr}\left(L^{2}\right)+\ddot{u}+\frac{\epsilon}{2} & =0, \\
r_{t}-(\operatorname{tr} L) L-\dot{L}+\dot{u} L+\frac{\epsilon}{2} \mathbb{I} & =0 .
\end{aligned}
$$

We have a conservation law

$$
\ddot{u}+(\operatorname{tr} L) \dot{u}-(\dot{u})^{2}-\epsilon u=C
$$

for some constant $C$. Using the equations this may be rewritten as

$$
\operatorname{tr}\left(r_{t}\right)+\operatorname{tr}\left(L^{2}\right)-(\dot{u}-\operatorname{tr} L)^{2}-\epsilon u+\frac{1}{2}(n-1) \epsilon=C .
$$

We now specialise to the case of steady solitons, that is, $\epsilon=0$. The conservation law is now

$$
\operatorname{tr}\left(r_{t}\right)+\operatorname{tr}\left(L^{2}\right)-(\dot{u}-\operatorname{tr} L)^{2}=C .
$$

Proposition 1.6. The function $(\dot{u}-\operatorname{tr} L)^{-2}$ is a Lyapunov function, that is, it is monotonic on each interval on which it is defined.

Proof.

$$
\begin{aligned}
\frac{d}{d t}\left(\frac{1}{(\dot{u}-\operatorname{tr} L)^{2}}\right) & =-\frac{2(\ddot{u}-\operatorname{tr} \dot{L})}{(\dot{u}-\operatorname{tr} L)^{3}} \\
& =-\frac{2 \operatorname{tr}\left(L^{2}\right)}{(\dot{u}-\operatorname{tr} L)^{3}}
\end{aligned}
$$


Remark 1.7. The conservation law (1.5) shows that our Lyapunov function is a constant multiple of

$$
\frac{\operatorname{tr}\left(r_{t}\right)+\operatorname{tr}\left(L^{2}\right)}{(\dot{u}-\operatorname{tr} L)^{2}}-1 .
$$

Remark 1.8. Recall that the above conservation law was derived in [DW] from the consequence $\Delta d u=d u \circ$ Ric of the Ricci soliton equation upon application of the contracted second Bianchi identity. In fact, we can also derive this conservation law from Perelman's $\mathcal{F}$-functional (steady case) and $\mathcal{W}$-functional (expanding and contracting cases). From this point of view, the conservation law asserts that the trajectories representing smooth gradient Ricci solitons must lie in the zero-energy hypersurface of the Hamiltonians corresponding to Perelman's functionals. Details of the Hamiltonian formulation will be discussed elsewhere.

\section{Multiple warped products}

We now specialise to the class of examples, multiple warped products, that will generalise the examples of Bryant and Ivey.

We look for metrics of the form

$$
d t^{2}+\sum_{i=1}^{r} g_{i}^{2}(t) h_{i}
$$

on $I \times M_{1} \times \ldots \times M_{r}$ where $I$ is an interval in $\mathbb{R}$ and $\left(M_{i}, h_{i}\right)$ are Einstein manifolds with positive Einstein constants $\lambda_{i}$. We let $d_{i}$ denote the (real) dimension of $M_{i}$.

Recall that the soliton potential $u$ is taken to be a function of $t$ alone. The resulting equations are equivalent to those coming from a cohomogeneity one ansatz, though of course the $M_{i}$ could be inhomogeneous.

The shape operator and Ricci endomorphism are now given by

$$
\begin{aligned}
L_{t} & =\operatorname{diag}\left(\frac{\dot{g_{1}}}{g_{1}} \mathbb{I}_{d_{1}}, \cdots, \frac{\dot{g}_{r}}{g_{r}} \mathbb{I}_{d_{r}}\right) \\
r_{t} & =\operatorname{diag}\left(\frac{\lambda_{1}}{g_{1}^{2}} \mathbb{I}_{d_{1}}, \cdots, \frac{\lambda_{r}}{g_{r}^{2}} \mathbb{I}_{d_{r}}\right)
\end{aligned}
$$

where $\mathbb{I}_{m}$ denotes the identity matrix of size $m$. (We shall henceforth drop the subscript $t$ of $L$ for ease of notation.) Motivated by Ivey's work, we introduce new variables

$$
\begin{aligned}
X_{i} & =\frac{\sqrt{d_{i}}}{(-\dot{u}+\operatorname{tr} L)} \frac{\dot{g}_{i}}{g_{i}} \\
Y_{i} & =\frac{\sqrt{d_{i} \lambda_{i}}}{g_{i}} \frac{1}{(-\dot{u}+\operatorname{tr} L)}
\end{aligned}
$$

for $i=1, \ldots, r$.

Notice that

$$
\sum_{j=1}^{r} X_{j}^{2}=\frac{\operatorname{tr}\left(L^{2}\right)}{(\dot{u}-\operatorname{tr} L)^{2}} \quad: \quad \sum_{j=1}^{r} Y_{j}^{2}=\frac{\operatorname{tr}\left(r_{t}\right)}{(\dot{u}-\operatorname{tr} L)^{2}} .
$$


We can take our Lyapunov function, therefore, to be

$$
\mathcal{L}:=\frac{C}{(\dot{u}-\operatorname{tr} L)^{2}}=\sum_{i=1}^{r}\left(X_{i}^{2}+Y_{i}^{2}\right)-1
$$

where $C$ is a nonzero constant to be specified later. Note further that

$$
\mathcal{L}=\tilde{C}_{i} g_{i}^{2} Y_{i}^{2}: i=1, \ldots, r
$$

for nonzero constants $\tilde{C}_{i}=\frac{C}{d_{i} \lambda_{i}}$.

It is convenient to introduce a new independent variable $s$ defined by

$$
\frac{d}{d s}:=\frac{1}{(-\dot{u}+\operatorname{tr} L)} \frac{d}{d t}=\sqrt{\frac{\mathcal{L}}{C}} \frac{d}{d t}=\frac{g_{i} Y_{i}}{\sqrt{\lambda_{i} d_{i}}} \frac{d}{d t},
$$

in which the final expression is independent of $i$ by (2.5). We use a prime ' to denote differentiation with respect to $s$.

We obtain from the Ricci soliton system the following equations in our new variables:

$$
\begin{aligned}
X_{i}^{\prime} & =X_{i}\left(\sum_{j=1}^{r} X_{j}^{2}-1\right)+\frac{Y_{i}^{2}}{\sqrt{d_{i}}}, \\
Y_{i}^{\prime} & =Y_{i}\left(\sum_{j=1}^{r} X_{j}^{2}-\frac{X_{i}}{\sqrt{d_{i}}}\right)
\end{aligned}
$$

for $i=1, \ldots, r$. Note that these imply the equation

$$
\mathcal{L}^{\prime}=2 \mathcal{L}\left(\sum_{i=1}^{r} X_{i}^{2}\right) .
$$

Conversely, if we have a solution of the above system, we may recover $t$ and the $g_{i}$ from

$$
d t=\sqrt{\frac{\mathcal{L}}{C}} d s, \quad g_{i}=\sqrt{\frac{\mathcal{L}}{C}} \frac{\sqrt{d_{i} \lambda_{i}}}{Y_{i}},
$$

which are equivalent to (2.6) and (2.3) respectively. The soliton potential is recovered from integrating

$$
\dot{u}=\operatorname{tr}(L)-\sqrt{\frac{C}{\mathcal{L}}}
$$

where $\operatorname{tr}(L)$ is calculated using

$$
\frac{\dot{g}_{i}}{g_{i}}=\sqrt{\frac{C}{\mathcal{L}}} \frac{X_{i}}{\sqrt{d_{i}}} .
$$

Differentiating the above, which is equivalent to (2.2), one gets

$$
\frac{\ddot{g}_{i}}{g_{i}}=\frac{C}{\mathcal{L}}\left(\frac{X_{i}^{2}+Y_{i}^{2}-\sqrt{d_{i}} X_{i}}{d_{i}}\right) .
$$

Putting (2.11)-(2.13) together one obtains (1.3). Finally, differentiating (2.11) gives (1.2). We then obtain a smooth solution of the Ricci soliton equation provided that 
appropriate smoothness conditions at the endpoints (formulated in the next section) are satisfied.

Remark 2.14. In (2.11) if we take the derivative with respect to $s$ instead, we have

$$
u^{\prime}=\frac{\operatorname{tr} L}{\sqrt{C / \mathcal{L}}}-1=\sum_{i=1}^{r} \sqrt{d_{i}} X_{i}-1,
$$

where we have used (2.12). Observe that $u^{\prime} \equiv 0$ iff the soliton is trivial (Ricci-flat in the steady case). This motivates the definition of the quantity $\mathcal{H}:=\sum_{i=1}^{r} \sqrt{d_{i}} X_{i}$, so that the Ricci-flat trajectories lie in the subspace $\mathcal{H}=1$. For these trajectories the conservation law (1.5) becomes $\mathcal{L}=0$.

\section{Trajectories of the equations}

Recall that by applying the maximum principle to consequences of the Ricci soliton equation one can show that on a closed manifold a steady Ricci soliton is Ricciflat (see e.g. Proposition 1.66 in [Cetc]). Hence we are interested in constructing complete non-compact steady soliton metrics where there is a smooth collapse at one end, corresponding to $t=0$ without any loss of generality, onto a lower-dimensional submanifold. This can be achieved if we take one factor, say $M_{1}$, to be a sphere $S^{d_{1}}$. The submanifold would then be $M_{2} \times \cdots \times M_{r}$. With the normalization $\lambda_{1}=d_{1}-1$, the boundary conditions for the soliton solution to be $C^{2}$ are the existence of the following limits:

$$
\begin{gathered}
g_{1}(0)=0: g_{i}(0)=l_{i} \neq 0(i>1), \\
\dot{g}_{1}(0)=1: \dot{g}_{i}(0)=0(i>1), \\
\ddot{g}_{1}(0)=0: \ddot{g}_{i}(0) \text { finite }(i>1), \\
u(0) \text { finite }: \dot{u}(0)=0: \ddot{u}(0) \text { finite. }
\end{gathered}
$$

In order to get a smooth solution, it suffices to show further that the third derivatives of $g_{i}$ tend to finite limits at $t=0$. Once this is done, we can write the trace of the soliton equation (1.1) as

$$
\Delta u=R+\frac{n \epsilon}{2} .
$$

The right-hand side of this elliptic equation for $u$ lies in $C^{0, \alpha}$. Since $u$ is in $C^{2}$, it follows from Lemma 6.16 in $[\mathrm{GT}]$ that $u$ is in $C^{2, \alpha}$. Using the contracted second Bianchi identity and the weak form of Bochner's formula for the Laplacian of a oneform (verified for example by smooth approximation in $W^{1,2}$ ) we can then show that the 1 -form $\omega:=d u$ is a weak solution of $\Delta \omega=2 \omega \circ r$ (Eq. (2.1) in [DW]). The argument in the proof of Lemma 2.2 there shows that $\omega$ is actually in $C^{2, \alpha}$. The smoothness (in fact real analyticity) of the solution then follows from Morrey's theorem. 
With the above remarks in mind, we consider trajectories emanating from the critical point of (2.7) and (2.8) given by

$$
X_{1}=\beta, \quad Y_{1}=\hat{\beta}, \quad X_{i}=Y_{i}=0(i>1)
$$

where $\beta=\frac{1}{\sqrt{d_{1}}}$ and $\hat{\beta}=+\sqrt{1-\beta^{2}}$. This critical point lies on the unit sphere in $X Y$-space, i.e., on the level set $\mathcal{L}=0$.

Linearising about this critical point gives a system whose matrix has a $2 \times 2$ block

$$
\left(\begin{array}{cc}
3 \beta^{2}-1 & 2 \beta \hat{\beta} \\
\beta \hat{\beta} & 0
\end{array}\right)
$$

corresponding to $X_{1}, Y_{1}$ : the remaining entries are diagonal, with $\beta^{2}$ and $\beta^{2}-1$ each occurring $r-1$ times. The eigenvalues are therefore $\beta^{2}(r-1$ times $), \beta^{2}-1$ ( $r$ times), and $2 \beta^{2}$.

We shall assume from now on that $d_{1}>1$. The above critical point is then hyperbolic.

We will parametrise trajectories emanating from this critical point so that the critical point corresponds to $s=-\infty$. Note that there is some $\delta>0$ such that for $i>1$, the differential inequality

$$
\frac{d}{d s} Y_{i}^{2} \leq 2\left(\beta^{2}+\delta\right) Y_{i}^{2}
$$

holds near $s=-\infty$ for any such trajectory. A comparison argument then shows that if $Y_{i}\left(s_{*}\right)>0$ for all $i>1$, then on $\left(-\infty, s_{*}\right]$ we have $Y_{i}>0$. Since $\lim _{s \rightarrow-\infty} Y_{1}=\hat{\beta}>0$ we may assume that $Y_{1}>0$ on $\left(-\infty, s_{*}\right]$ as well.

Now by standard facts in dynamical systems (cf [CL], proofs of Theorems 4.1, 4.3 and 4.5) and the fact that the system (2.7)-(2.8) is invariant under the symmetries $Y_{i} \mapsto-Y_{i}$, there is an $(r-1)$-parameter family of trajectories lying in the unstable manifold of this critical point having the above positivity properties and flowing into the open unit ball $\mathcal{L}<0$.

Remark 3.5. In [Bo] an $r-2$ parameter family of complete Ricci-flat metrics was constructed on the manifolds under consideration here. These correspond to trajectories emanating from the above critical point and lying in the sphere $\mathcal{L}=0$. In fact, the unstable manifold intersects this sphere transversely, and this accounts for the parameters in the Ricci-flat metrics.

We now work with one of the trajectories going into $\mathcal{L}<0$.

Eq. (2.9) shows that the trajectory stays in the region $\mathcal{L}<0$. Hence all the variables are bounded by 1 and the flow exists for all $s \in \mathbb{R}$. Moreover, $\mathcal{L}$ decreases monotonically to some negative constant $\kappa$. Note that as a result of the above choices, the constant $C$ in (2.4) is fixed and is negative.

Now Eq. (2.8) and $X_{i} \leq 1$ imply that $Y_{i}^{2}$ satisfies the differential inequality

$$
\frac{d}{d s} Y_{i}^{2} \geq-\frac{2}{\sqrt{d_{i}}} Y_{i}^{2} .
$$

By a standard comparison argument it follows from $Y_{i}\left(s_{*}\right)>0$ that $Y_{i}>0$ on $\left[s_{*},+\infty\right)$. Hence for all finite $s, g_{i}$ can be defined by (2.10) (so is nonzero) and (2.5) holds. 
Lemma 3.6. The metric corresponding to our trajectory is complete at $s=+\infty$.

Proof. From (2.10) it follows that as $s$ tends to infinity the arclength $t$ does also. The definition of $g_{i}$ shows that $g_{i}$ remain nonzero on $s>s_{*}$.

We can refine our analysis to study the asymptotics of the metric as $s$ tends to $+\infty$.

Proposition 3.7. The trajectory converges to the origin as s tends to $+\infty$.

Proof. Recall that the $\omega$-limit set of the trajectory is the set

$$
\Omega=\left\{\left(X^{*}, Y^{*}\right): \exists s_{n} \rightarrow+\infty \text { with }\left(X\left(s_{n}\right), Y\left(s_{n}\right)\right) \rightarrow\left(X^{*}, Y^{*}\right)\right\} .
$$

As our trajectory ultimately lies in a compact set, we know from standard theory $([\mathrm{Pk}] \S 3.2)$ that $\Omega$ is a compact, connected, non-empty set that is invariant under the flow of our equations. Moreover, $\Omega$ is contained in the sphere $\mathcal{L}=\kappa$.

Now if $\Omega$ contains a point $\left(X^{*}, Y^{*}\right)$ with $X^{*} \neq 0$, we see from $(2.9)$ that $\mathcal{L}^{\prime}$ at this point is nonzero, contradicting the flow-invariance of $\Omega$. Hence $\Omega$ is contained in the set $X_{i}=0(i=1, \ldots, r)$. Furthermore, if $\Omega$ contains a point $\left(0, Y^{*}\right)$ with $Y^{*} \neq 0$, we see from (2.7) that some $X_{i}^{\prime}$ is nonzero, again contradicting flow-invariance.

Hence $\Omega=(0,0)$, and the limiting value $\kappa$ of $\mathcal{L}$ is -1 , showing that the trajectory does indeed converge to the origin.

Lemma 3.8. We have $\lim _{s \rightarrow \infty} \frac{X_{i}}{Y_{i}^{2}}=\frac{1}{\sqrt{d_{i}}}$.

Proof. Observe that $X_{i} / Y_{i}^{2}$ satisfies the differential equation

$$
\left(\frac{X_{i}}{Y_{i}^{2}}\right)^{\prime}=\left(-1-\sum_{j=1}^{r} X_{j}^{2}+\frac{2 X_{i}}{\sqrt{d_{i}}}\right) \frac{X_{i}}{Y_{i}^{2}}+\frac{1}{\sqrt{d_{i}}} .
$$

By Prop 3.7, the coefficient of $\frac{X_{i}}{Y_{i}^{2}}$ tends to -1 as $s \rightarrow+\infty$. In particular, if $\frac{X_{i}}{Y_{i}^{2}}$ tends to a limit $a$, then its derivative tends to $-a+\frac{1}{\sqrt{d_{i}}}$, so $a$ must equal $\frac{1}{\sqrt{d_{i}}}$.

Let $0<\delta<1$, and pick $s^{*}(\delta)$ so that the absolute value of $-\sum_{j=1}^{r} X_{j}^{2}+\frac{2 X_{i}}{\sqrt{d_{i}}}$ is less than $\delta$ for $s>s^{*}(\delta)$. It follows that if $\frac{X_{i}}{Y_{i}^{2}}\left(s_{0}\right) \geq \frac{1}{\sqrt{d_{i}}(1-\delta)}$ for some $s_{0}>s^{*}(\delta)$, then $\left(\frac{X_{i}}{Y_{i}^{2}}\right)^{\prime}<0$ at $s_{0}$. Similarly, if $\frac{X_{i}}{Y_{i}^{2}}\left(s_{0}\right) \leq \frac{1}{\sqrt{d_{i}}(1+\delta)}$, then $\left(\frac{X_{i}}{Y_{i}^{2}}\right)^{\prime}>0$ at $s_{0}$.

So if $\frac{X_{i}}{Y_{i}^{2}}$ enters the horizontal strip $\frac{1}{\sqrt{d_{i}}(1+\delta)}<y<\frac{1}{\sqrt{d_{i}}(1-\delta)}$ at some $s>s^{*}(\delta)$ it is trapped there. Hence one of the following must hold:

(i) $\frac{X_{i}}{Y_{i}^{2}}(s) \geq \frac{1}{\sqrt{d_{i}}(1-\delta)}$ for all $s>s^{*}(\delta)$,

(ii) $\frac{X_{i}}{Y_{i}^{2}}(s) \leq \frac{1}{\sqrt{d_{i}}(1+\delta)}$ for all $s>s^{*}(\delta)$, or

(iii) $\frac{1}{\sqrt{d_{i}}(1+\delta)}<\frac{X_{i}}{Y_{i}^{2}}<\frac{1}{\sqrt{d_{i}}(1-\delta)}$ for $s$ sufficiently large.

In Case (i), $\frac{X_{i}}{Y_{i}^{2}}$ is monotonic decreasing and bounded below by $\frac{1}{\sqrt{d_{i}}(1-\delta)}$, so it tends to a finite limit which must be at least $\frac{1}{\sqrt{d_{i}}(1-\delta)}>\frac{1}{\sqrt{d_{i}}}$, contradicting the discussion above. Case (ii) is eliminated similarly.

The remaining possibility is that (iii) holds for all $\delta$; hence $\frac{X_{i}}{Y_{i}^{2}} \rightarrow \frac{1}{\sqrt{d_{i}}}$ as claimed. 
We deduce that $g_{i} \dot{g}_{i}$ asymptotically approaches the constant $\lambda_{i} / \sqrt{-C}$, and obtain the following theorem.

Theorem 3.10. The metric corresponding to our trajectory is, to leading order in $t$ as $t \rightarrow+\infty$,

$$
d t^{2}+t \mathrm{~h}_{\infty}
$$

where the homothety class of $\mathrm{h}_{\infty}$ is that of the product Einstein metric on $M_{1} \times \cdots \times$ $M_{r}$. So our metric has an asymptotically paraboloid geometry.

Remark 3.11. The Bryant solitons on $\mathbb{R}^{n}$ (for $n>2$ ) also have asymptotically paraboloid geometry. On the other hand the complete steady Kähler solitons considered in $[\mathrm{DW}]$ are asymptotically circle bundles of constant radius over paraboloids.

These two kinds of asymptotics may be viewed as the Ricci soliton analogues of the Asymptotically Conical (AC) and Asymptotically Locally Conical (ALC) conditions satisfied by many of the known complete non-compact Ricci-flat metrics (see [CGLP] for example).

\section{Analysing the flow}

To check smoothness at the collapsing submanifold, we must now analyse the trajectory as $s$ tends to $-\infty$. Recall that $X_{1} \rightarrow \beta=\frac{1}{\sqrt{d_{i}}}, Y_{1} \rightarrow+\sqrt{1-\beta^{2}}$ and the remaining variables tend to 0 .

Remark 4.1. Observe from $(2.9)$ that $\mathcal{L}$ (and hence $g_{i}^{2} Y_{i}^{2}$ ) tend to zero exponentially fast as $s$ tends to $-\infty$.

The following lemma is often useful.

Lemma 4.2. Suppose a function $F$ satisfies a differential equation

$$
F^{\prime}=H F+K
$$

where $H, K$ are functions tending respectively to finite limits $h, k$ as $s$ tends to $-\infty$, where $h<0$ and $k \neq 0$.

Then either $\lim _{s \rightarrow-\infty} F(s)=-\frac{k}{h}$ or $F$ tends to $\infty$ or $-\infty$ as $s$ tends to $-\infty$. Moreover in the case of infinite limit $F$ is monotonic for sufficiently large negative $s$.

Proof. We give the proof for the case $k>0$ below; obvious modifications yield the proof for the case $k<0$.

Let $0<\delta<\min (-h, k)$, and choose $s^{*}(\delta)$ so that for all $s \leq s^{*}(\delta)$ we have

$$
h-\delta<H(s)<h+\delta<0: 0<k-\delta<K(s)<k+\delta \text {. }
$$

If $F\left(s_{0}\right) \leq \frac{k-\delta}{-h+\delta}$ for some $s_{0} \leq s^{*}(\delta)$, then $F^{\prime}\left(s_{0}\right)>0$. Hence these inequalities for $F, F^{\prime}$ actually hold for all $s \leq s_{0}$. So as $s$ tends to $-\infty$, either $F$ tends to $-\infty$ (monotonically on $\left(-\infty, s_{0}\right]$ ) or to a finite limit $\xi<\frac{k-\delta}{-h+\delta}<-\frac{k}{h}$. But in the latter case $F^{\prime}$ tends to a nonzero limit, which is impossible.

If $F\left(s_{0}\right) \geq \frac{k+\delta}{-h-\delta}$, then we similarly see that $F$ tends monotonically to $+\infty$ as $s$ tends to $-\infty$.

So if $F$ does not tend monotonically to $\pm \infty$, we see that for all such $\delta$ we have $\frac{k-\delta}{-h+\delta}<F(s)<\frac{k+\delta}{-h-\delta}$ on $\left(-\infty, s^{*}(\delta)\right]$. Hence $F$ tends to $-\frac{k}{h}$ as $s$ tends to $-\infty$. 
Lemma 4.4. The function $X_{i} / Y_{i}^{2}$ is positive, and remains bounded as $s$ tends to $-\infty$.

Proof. (i) Positivity. Note that $\left(\sum_{j=1}^{r} X_{j}^{2}\right)-1$ is bounded above by $\mathcal{L}$ so it is negative. Now since $Y_{i}>0$ for all $s$, if $X_{i} \leq 0$ at some $s_{0}$, we see that $X_{i}^{\prime}$ is positive at $s_{0}$. Hence $X_{i}$ is negative and $X_{i}^{\prime}$ positive on $\left(-\infty, s_{0}\right)$, which contradicts the fact that $\lim _{s \rightarrow-\infty} X_{i}=0$ (if $i>1$ ), or $\beta=\frac{1}{\sqrt{d_{1}}}>0$ if $i=1$. We deduce that $X_{i}$ is positive for all finite $s$.

(ii) Boundedness. This is trivial if $i=1$, so in what follows we take $i>1$. We know that $\left(\sum_{i=1}^{r} X_{j}^{2}\right)-1$ tends to $\beta^{2}-1$ and $\left(\sum_{j=1}^{r} X_{j}^{2}\right)-\frac{X_{i}}{\sqrt{d_{i}}}$ tends to $\beta^{2}$ as $s$ tends to $-\infty$. Pick $s^{*}$ so that $\left(\sum_{j=1}^{r} X_{j}^{2}\right)-1<\frac{1}{2}\left(\beta^{2}-1\right)<0$ and $\left(\sum_{j=1}^{r} X_{j}^{2}\right)-\frac{X_{i}}{\sqrt{d_{i}}}>\frac{1}{2} \beta^{2}>0$ for $s \leq s^{*}$. Note that this implies that $Y_{i}^{\prime}$ is positive on $\left(-\infty, s^{*}\right]$, since, as discussed in $\S 3$, we can take the $Y_{i}$ to be positive.

Suppose that $\frac{X_{i}}{Y_{i}^{2}}>\frac{2}{\sqrt{d_{i}\left(1-\beta^{2}\right)}}$ at some $s_{0} \leq s^{*}$. It follows from our choice of $s^{*}$ that $X_{i}^{\prime}$ is negative at $s_{0}$. As remarked above, $Y_{i}$ and $Y_{i}^{\prime}$ are positive on $\left(-\infty, s^{*}\right]$. It follows that these inequalities for $X_{i} / Y_{i}^{2}$ and the derivatives of $X_{i}, Y_{i}$ actually hold on $\left(-\infty, s_{0}\right]$. But this contradicts the fact that $X_{i}$ tends to zero as $s$ tends to $-\infty$. So we have the desired bound on $X_{i} / Y_{i}^{2}$.

Proposition 4.5. For $i>1$, we have

$$
\lim _{s \rightarrow-\infty} \frac{X_{i}}{Y_{i}^{2}}=\frac{1}{\sqrt{d_{i}}\left(1+\beta^{2}\right)} .
$$

Proof. The differential equation (3.9) is of the form (4.3), with $h=-\left(1+\beta^{2}\right)$ and $k=\frac{1}{\sqrt{d_{i}}}$. The desired result now follows from Lemma 4.2 and Lemma 4.4.

Corollary 4.6. As $s$ tends to $-\infty$, the arclength $t$ can be chosen to tend to zero. Moreover, we have the following limiting values for $g_{i}(t)$ as $t$ tends to 0 .

$$
g_{1}(0)=0 \quad: \quad \dot{g}_{1}(0)=1 \quad: \quad \dot{g}_{i}(0)=0(i>1) .
$$

Proof. The first statement follows from Remark 4.1 and equation (2.6). The statement about $g_{1}$ comes from (2.10) using this Remark and the fact that $Y_{1}$ tends to a nonzero value as $s$ tends to $-\infty$.

Since $\dot{g}_{i}=\sqrt{\lambda_{i}}\left(X_{i} / Y_{i}\right)$, the remaining limits follow from Lemma 4.4, the known limits of $X_{i}, Y_{i}$, and the fact that $\lambda_{1}=d_{1}-1$.

Proposition 4.7. For $i>1, g_{i}(0)$ is finite and nonzero.

Proof. Observe that $\frac{d}{d s}\left(g_{i}^{2}\right)=2 g_{i}^{2} X_{i} / \sqrt{d_{i}}$, which is positive for all $s$. So $g_{i}^{2}$ tends to a finite, nonnegative limit at $s=-\infty$.

To get positivity, we consider

$$
\frac{g_{i}^{\prime}}{g_{i}}=\frac{X_{i}}{\sqrt{d_{i}}}=\frac{1}{2 \sqrt{d_{i}}} \frac{X_{i}}{Y_{i}^{2}} \frac{\left(Y_{i}^{2}\right)^{\prime}}{\left(\sum_{j=1}^{r} X_{j}^{2}\right)-\frac{X_{i}}{\sqrt{d_{i}}}} .
$$

Integrating, using Prop. 4.5, and observing that the denominator in the last factor tends to $\beta^{2}$, we get a bound

$$
\frac{g_{i}\left(s^{*}\right)}{g_{i}(s)} \leq \exp \left(\frac{Y_{i}^{2}\left(s^{*}\right)-Y_{i}^{2}(s)}{2 d_{i}\left(\beta^{2}-\delta\right)\left(1+\beta^{2}-\delta\right)}\right)
$$


(for some positive $\delta$ ), giving the desired positive lower bound on $g_{i}(s)$.

We shall next obtain some estimates that will be useful for studying the second derivatives of $g_{i}$ and $u$.

Lemma 4.8. We have $X_{1}<\beta$ for all finite $s$.

Proof. We can rewrite the equation (2.7) for $X_{1}$ as

$$
X_{1}^{\prime}=\left(X_{1}-\beta\right)\left(\mathcal{L}-\sum_{j=1}^{r} Y_{j}^{2}\right)+\beta\left(\mathcal{L}-\sum_{j=2}^{r} Y_{j}^{2}\right) .
$$

The terms in the second and third brackets are negative for finite $s$, since $\mathcal{L}$ is. Hence if $X_{1} \geq \beta$ at some $s_{0}$ we see $X_{1}^{\prime}$ is negative at $s_{0}$, and hence these inequalities hold on $\left(-\infty, s_{0}\right]$. It follows that $X_{1}$ cannot tend to $\beta$ as $s$ tends to $-\infty$, a contradiction.

Recalling that $\mathcal{L}$ is negative on our trajectory, this shows that $\frac{X_{1}-\beta}{\mathcal{L}}$ is positive.

We note next that $\left(X_{1}-\beta\right) / \mathcal{L}$ satisfies the differential equation

$$
\left(\frac{X_{1}-\beta}{\mathcal{L}}\right)^{\prime}=\left(\frac{X_{1}-\beta}{\mathcal{L}}\right)\left(-1-\sum_{j=1}^{r} X_{j}^{2}\right)+\beta\left(\frac{\sum_{j=1}^{r} X_{j}^{2}+Y_{1}^{2}-1}{\mathcal{L}}\right) .
$$

Observe also that

$$
\rho:=\lim _{s \rightarrow-\infty} \frac{\sum_{j=1}^{r} X_{j}^{2}+Y_{1}^{2}-1}{\mathcal{L}}
$$

exists and is a finite number greater than 1 , because the numerator is $\mathcal{L}-\sum_{j=2}^{r} Y_{j}^{2}$, and we know for $j>1$ that $Y_{j}^{2} / \mathcal{L}$ tends to a finite negative limit as $s$ tends to $-\infty$ (as this is a negative constant times $g_{j}^{-2}$ ).

Now Lemma 4.2 shows that as $s$ tends to $-\infty, \frac{X_{1}-\beta}{\mathcal{L}}$ either tends to infinity or tends to the positive limit $\frac{\beta \rho}{1+\beta^{2}}$. Moreover, because it cannot tend to $-\infty$, Lemma 4.2 gives a positive lower bound on $\frac{X_{1}-\beta}{\mathcal{L}}$.

Remark 4.9. We can make some statements about the decay rates of our variables as $s$ tends to $-\infty$. From (2.8) we see that for $i>1$ we have $Y_{i} \sim e^{\beta^{2} s}$, hence, by Prop. 4.5 , we also have $X_{i} \sim e^{2 \beta^{2} s}$. It follows from (2.9) that $\mathcal{L} \sim e^{2 \beta^{2} s}$. Now the remarks in the previous paragraph show that $X_{1}-\beta$ decays more slowly than $e^{\left(2 \beta^{2}+\delta\right) s}$, for any positive $\delta$.

Lemma 4.10. The quantity $\frac{X_{1}-\beta}{\mathcal{L}}$ cannot tend to $\infty$ as $s$ tends to $-\infty$.

Proof. Observe that

$$
\frac{\left(Y_{1}-\hat{\beta}\right)^{\prime}}{\mathcal{L}^{\prime}}=\frac{Y_{1}}{2 \sum_{j=1}^{r} X_{j}^{2}}\left(\frac{\sum_{j=2}^{r} X_{j}^{2}}{\mathcal{L}}+\frac{X_{1}\left(X_{1}-\beta\right)}{\mathcal{L}}\right) .
$$

Now the term outside the bracket tends to a nonzero finite limit, and the first term in the bracket tends to zero. So if $\frac{X_{1}-\beta}{\mathcal{L}}$ tends to $+\infty$, so does $\frac{\left(Y_{1}-\hat{\beta}\right)^{\prime}}{\mathcal{L}^{\prime}}$, and hence, by L'Hôpital's rule, so does $\frac{Y_{1}-\hat{\beta}}{\mathcal{L}}$. But

$\frac{\sum_{j=1}^{r} X_{j}^{2}+Y_{1}^{2}-1}{\mathcal{L}}=\frac{2 \beta\left(X_{1}-\beta\right)+2 \hat{\beta}\left(Y_{1}-\hat{\beta}\right)+\left(X_{1}-\beta\right)^{2}+\left(Y_{1}-\hat{\beta}\right)^{2}+\sum_{j=2}^{r} X_{j}^{2}}{\mathcal{L}}$ 
The discussion in Remark 4.9 shows the first two terms are the dominant ones, so we deduce the expression on the left-hand-side tends to $+\infty$, which we saw above is false.

We now have

\section{Proposition 4.12.}

$$
\lim _{s \rightarrow-\infty}\left(\frac{X_{1}-\beta}{\mathcal{L}}\right)=\frac{\beta \rho}{1+\beta^{2}}
$$

Proposition 4.13. As $s$ tends to $-\infty, e^{-2 \beta^{2} s} \mathcal{L}$ tends to a finite negative limit.

Proof. Letting $F=e^{-2 \beta^{2} s} \mathcal{L}$, (so that $F$ is negative), we see that

$$
F^{\prime}=2 F\left(\left(\sum_{j=1}^{r} X_{j}^{2}\right)-\beta^{2}\right) .
$$

As in the proof of Lemma 4.10, we see that the dominant term in the bracket is $2 \beta\left(X_{1}-\beta\right)$, which is negative by Lemma 4.8. So $F$ is monotonic increasing for large negative $s$.

The proposition is proved if we can show that $F$ is bounded below near $s=-\infty$. This follows by estimating the integral of the right-hand side of the above equation over an interval $\left(-\infty, s^{*}\right]$ on which we have bounds of the form

$$
\begin{gathered}
\left|X_{1}-\beta\right| \leq A \exp \left(2\left(\beta^{2}-\delta\right) s\right), \quad\left|X_{j}(s)\right| \leq C_{j} \exp \left(2\left(\beta^{2}-\delta\right) s\right) \\
\left|X_{1}+\beta\right| \leq 2 \beta+\delta, \quad|\mathcal{L}| \leq B \exp \left(2\left(\beta^{2}-\delta\right) s\right)
\end{gathered}
$$

where $\delta, A, B, C_{j}, 2 \leq j \leq r$ are appropriate positive constants (cf Remark 4.9).

We can now check the second derivatives of $g_{i}$ at $t=0$. Recall that from (2.13) we have

$$
\ddot{g}_{i}=\frac{\lambda_{i}}{g_{i} Y_{i}^{2}}\left(X_{i}^{2}+Y_{i}^{2}-\sqrt{d_{i}} X_{i}\right) .
$$

It is now clear from Prop 4.5 and Prop 4.7 that for $i>1, \ddot{g}_{i}$ tends to a finite limit as $t$ tends to zero.

If $i=1$, we rewrite this expression, using (2.3)-(2.5), as

$$
\ddot{g}_{1}=\frac{\lambda_{1}}{Y_{1}}\left(\frac{1-\sqrt{d_{1}} X_{1}}{g_{1} Y_{1}}\right)+g_{1}\left(\frac{C}{d_{1}}-\sum_{j=2}^{r} \frac{d_{j} \lambda_{j}}{d_{1}} \frac{1}{g_{j}^{2}}\left(\frac{X_{j}^{2}}{Y_{j}^{2}}+1\right)\right) .
$$

The quantities in the bracket after $g_{1}$ tend to finite limits, so $g_{1}$ times that bracket tends to zero. As $Y_{1}$ tends to $\hat{\beta} \neq 0$, we see from Prop 4.12 and (2.5) that the first term also tends to zero.

We have therefore shown the metric is $C^{2}$.

Similarly we can study the potential $u$. From the relation (2.11) we obtain

$$
\dot{u}=\sqrt{\frac{C}{\mathcal{L}}}\left(\left(\sum_{j=1}^{r} \sqrt{d_{j}} X_{j}\right)-1\right)=\sqrt{d_{1}}\left(\frac{\left(X_{1}-\beta\right)}{\sqrt{\mathcal{L} / C}}\right)+\sum_{i=2}^{r} \sqrt{d_{i}}\left(\frac{X_{i}}{Y_{i}^{2}}\right)\left(\frac{Y_{i}^{2}}{\sqrt{\mathcal{L} / C}}\right) .
$$


By Prop 4.5, Remark 4.9, and Prop 4.12, it follows that $\dot{u}$ tends to zero as $t$ tends to 0 . Next, by integrating (2.11) we get

$$
e^{u(0)}=(\text { positive constant }) \prod_{i=2}^{r} g_{i}(0)^{d_{i}}\left(\lim _{s \rightarrow-\infty} e^{-2 \beta^{2} s} \frac{\mathcal{L}}{C}\right)^{\frac{d_{1}}{2}},
$$

which is finite by Prop 4.13. If we differentiate (2.11) and use (2.9) we get

$$
\ddot{u}=\sum_{i=1}^{r} d_{i} \frac{\ddot{g}_{i}}{g_{i}}=\sum_{i=1}^{r} \frac{\lambda_{i} d_{i}}{g_{i}^{2} Y_{i}^{2}}\left(X_{i}^{2}+Y_{i}^{2}-\sqrt{d_{i}} X_{i}\right) .
$$

The right-hand side tends to a finite limit (as $t$ tends to 0 ) by Props 4.7, 4.12, the relation (2.5) and the discussion after Prop 4.13.

For the third derivatives, we calculate

$$
\frac{d^{3} g_{i}}{d t^{3}}=\sqrt{\frac{C}{\mathcal{L}}} \frac{\lambda_{i}}{g_{i}}\left(\frac{X_{i}}{Y_{i}^{2}}\left(-3 X_{i}+\frac{X_{i}^{2}}{\sqrt{d_{i}}}+\sqrt{d_{i}}+\sqrt{d_{i}} \sum_{j=1}^{r} X_{j}^{2}\right)+\frac{X_{i}}{\sqrt{d_{i}}}-1\right) .
$$

If $i>1$, we know that the terms in $\frac{X_{i}}{\sqrt{\mathcal{L}}}, \frac{X_{i}^{2}}{Y_{i}^{2} \sqrt{\mathcal{L}}}, \frac{X_{i}^{3}}{Y_{i}^{2} \sqrt{\mathcal{L}}}$ tend to zero (cf Remark 4.9). Our task thus reduces to showing that

$$
\frac{1}{\sqrt{\mathcal{L}}}\left(\frac{X_{i}}{Y_{i}^{2}}\left(1+\sum_{j=1}^{r} X_{j}^{2}\right)-\frac{1}{\sqrt{d_{i}}}\right)
$$

tends to zero.

Now, $1+\sum_{j=1}^{r} X_{j}^{2}=1+\beta^{2}$ modulo terms approaching zero at least as fast as $\mathcal{L}$, so we just have to check that

$$
\lim _{s \rightarrow-\infty} \frac{1}{\sqrt{\mathcal{L}}}\left(\frac{X_{i}}{Y_{i}^{2}}-\frac{1}{\sqrt{d_{i}}\left(1+\beta^{2}\right)}\right)=0 .
$$

In fact we shall show the stronger statement that

$$
Q_{i}:=\frac{1}{\mathcal{L}}\left(\frac{X_{i}}{Y_{i}^{2}}-\frac{1}{\sqrt{d_{i}}\left(1+\beta^{2}\right)}\right)
$$

tends to a finite limit. We find that $Q_{i}$ satisfies the equation

$$
Q_{i}^{\prime}=-\left(1+3 \sum_{j=1}^{r} X_{j}^{2}\right) Q_{i}+\frac{1}{\left(\sqrt{d_{i}}\left(1+\beta^{2}\right)\right) \mathcal{L}}\left(2\left(1+\beta^{2}\right) \frac{X_{i}^{2}}{Y_{i}^{2}}+\beta^{2}-\sum_{j=1}^{r} X_{j}^{2}\right) .
$$

By arguments similar to those above, one sees that the second term on the right-hand side tends to a finite negative limit as $s \rightarrow-\infty$ since $X_{1}<\beta$ and $\mathcal{L}<0$. So the hypotheses of Lemma 4.2 are satisfied, and $Q_{i}$ either tends to a finite limit or to $+\infty$ or $-\infty$. But

$$
\frac{\left(\frac{X_{i}}{Y_{i}^{2}}-\frac{1}{\sqrt{d_{i}}\left(1+\beta^{2}\right)}\right)^{\prime}}{\mathcal{L}^{\prime}}=\frac{\left(\frac{X_{i}}{Y_{i}^{2}}-\frac{1}{\sqrt{d_{i}}\left(1+\beta^{2}\right)}\right)}{\mathcal{L}}\left(\frac{-\left(1+\beta^{2}\right)}{2 \sum_{j=1}^{r} X_{j}^{2}}\right)+R_{i}
$$

where $R_{i}$ tends to a finite limit. So if the limit of $Q_{i}$ is infinite, L'Hôpital's rule gives a contradiction, as the term in the final bracket is negative. 
For $i=1$, by $(2.10)$ and $Y_{1}(0)=\hat{\beta}$, it is enough to check that

$$
\frac{-3\left(\frac{X_{1}}{Y_{1}}\right)^{2}+\beta \frac{X_{1}^{3}}{Y_{1}^{2}}+\beta X_{1}-1+\frac{X_{1}}{\beta Y_{1}^{2}}\left(1+\sum_{j=1}^{r} X_{j}^{2}\right)}{\mathcal{L}}
$$

has a finite limit. Recall that $1+\sum_{j=1}^{r} X_{j}^{2}=1+\beta^{2}$ modulo terms which have a finite limit when divided by $\mathcal{L}$. Similarly $X_{1}^{k}=\beta^{k}$ modulo such terms. So we are left with checking that the limit of $\frac{Y_{1}^{2}-\hat{\beta}^{2}}{\mathcal{L}}$ is finite, which follows on applying L'Hôpital's rule, equation (4.11) and Prop. 4.12.

We have shown that the metric is $C^{3}$ and so by the discussion on regularity near the beginning of $\S 3$, the soliton is smooth.

Theorem 4.17. Let $M_{2}, \ldots, M_{r}$ be compact Einstein manifolds with positive scalar curvature. For $d_{1}>1$ there is an $r-1$ parameter family of complete smooth steady Ricci solitons on the trivial rank $d_{1}+1$ vector bundle over $M_{2} \times \ldots \times M_{r}$.

The examples of Ivey and Bryant have nonnegative Ricci curvature. This is also true for our more general examples.

Proposition 4.18. The soliton metrics have nonnegative Ricci curvature.

Proof. It is enough to show that the Ricci curvature is positive on the complement of the submanifold at $t=0$, i.e., on the finite part of the trajectory. The Hessian of $u$ is given by $\ddot{u}$ evaluated on directions normal to the hypersurface and by $\frac{\dot{u} \dot{g}_{i}}{g_{i}}$ evaluated on directions tangent to $M_{i}$. From the soliton equation (1.1) (with $\epsilon=0$ ), the formulae (4.15), (4.14), (4.16), (2.5) above for $\dot{u}, \ddot{u}$ and the fact, proved in Prop (4.7), that $\frac{\dot{g}_{i}}{g_{i}}$ is positive, it is enough to check that

$$
\mathcal{H}=\sum_{i=1}^{r} \sqrt{d_{i}} X_{i}
$$

introduced in Remark 2.14, satisfies $\mathcal{H}<1$ and $\mathcal{L}+1-\mathcal{H}<0$ for all $s$.

Now it is easy to check that we have equations

$$
(\mathcal{H}-1)^{\prime}=(\mathcal{H}-1)\left(\sum_{j=1}^{r} X_{j}^{2}-1\right)+\mathcal{L}
$$

and

$$
(\mathcal{L}+1-\mathcal{H})^{\prime}=(\mathcal{L}+1-\mathcal{H})\left(\sum_{j=1}^{r} X_{j}^{2}-1\right)+\mathcal{L}\left(\sum_{j=1}^{r} X_{j}^{2}\right) .
$$

Moreover $\sum_{j=1} X_{j}^{2}-1$ and $\mathcal{L}$ are negative. So if $\mathcal{H} \geq 1$ at $s_{0}$ then $\mathcal{H}^{\prime}<0$ and $\mathcal{H}>1$ on $\left(-\infty, s_{0}\right)$, contradicting the fact that $\mathcal{H}$ tends to 1 as $s$ tends to $-\infty$. Similarly if $\mathcal{L}+1-\mathcal{H}$ is non-negative at $s_{0}$, then we see it is positive with negative derivative on $\left(-\infty, s_{0}\right)$, contradicting the fact that $\mathcal{L}+1-\mathcal{H}$ tends to zero.

Remark 4.19. For a multiply-warped product $I \times M_{1} \times \cdots \times M_{r}$ with metric of the form (2.1) it is easy to compute the sectional curvatures, e.g., by considering it as a 
Riemannian submersion over $I$. If $U, V$ are respectively tangent to $M_{i}$ and $M_{j}$, one has

$$
\begin{aligned}
K\left(U \wedge \frac{\partial}{\partial t}\right) & =-\frac{\ddot{g}_{i}}{g_{i}} \\
K(U \wedge V) & =-\frac{\dot{g}_{i} \dot{g}_{j}}{g_{i} g_{j}}, \quad i \neq j, \\
K(U \wedge V) & =\frac{1}{g_{i}^{2}}\left(K_{h_{i}}(U \wedge V)-\dot{g}_{i}^{2}\right), i=j,
\end{aligned}
$$

where $K_{h_{i}}$ denotes the sectional curvature of $\left(M_{i}, h_{i}\right)$.

It now follows from the asymptotics described in Theorem 3.10 that if $r>1$ there are always 2-planes with negative sectional curvature. If $r=1$, we are in the case of the Bryant solitons, which are known to have positive curvature ([Bry] or [Cetc Lemma 1.37]). The above formulas also show that the sectional curvatures decay like $t^{-1}$ as $t$ tends to $+\infty$. Recall that the asymptotic scalar curvature ratio of a complete, non-compact Riemannian manifold is defined as $\lim \sup _{d \rightarrow+\infty} R d^{2}$, where $R$ is the scalar curvature and $d$ is the distance from a fixed origin in the manifold. Since $t$ is the geodesic distance in our examples, it follows that their asymptotic scalar curvature ratios are all $+\infty$.

\section{Acknowledgements}

We would like to thank the referee for several helpful comments.

\section{References}

[ACGT] V. Apostolov, D. Calderbank, P. Gauduchon and C. Tønnesen-Friedman, Hamiltonian 2forms in Kähler Geometry IV: Weakly Bochner-Flat Kähler Manifolds, Comm. Anal. Geom. 16 (2008), 91-126.

[Bo] C. Böhm, Non-compact Cohomogeneity One Einstein Manifolds, Bull. Soc. Math. France, 122, (1999), 135-177.

[Bry] R. Bryant, unpublished work.

[Ca] H. D. Cao, Existence of Gradient Ricci Solitons, Elliptic and Parabolic Methods in Geometry, A. K. Peters, (1996), 1-16.

[CaZ] H. D. Cao and Xiping Zhu, A Complete Proof of the Poincaré and Geometrization Conjectures-Application of the Hamilton-Perelman Theory of the Ricci Flow, Asian J. Math., 10, (2006), 165-492.

[ChV] T. Chave and G. Valent, On a Class of Compact and Non-compact Quasi-Einstein Metrics and Their Renormalizability Properties, Nuclear Phys. B478, (1996), 758-778.

[Cetc] B. Chow, S.C. Chu, D. Glickenstein, C. Guenther, J. Isenberg, T. Ivey, D. Knopf, P. Lu, F. Luo, and L. Nei, The Ricci flow: Techniques and Applications Part I:Geometric Aspects, Mathematical Surveys and Monographs Vol. 135, American Math. Soc. (2007).

[CL] E. A. Coddington and N. Levinson, Theory of Ordinary Differential Equations, Reprint Edition, Krieger Publishing Co. Inc. (1984).

[CGLP] M. Cvetič, G. Gibbons, H. Lü and C. Pope, New complete noncompact Spin(7) manifolds, Nucl. Phys. B 620, (2002), 29-54.

[DW] A. Dancer and M. Wang, On Ricci Solitons of Cohomogeneity One, arXiv:math.DG/ 08020759 .

[DTK] D. DeTurck and J. Kazdan, Some Regularity Theorems in Riemannian Geometry, Ann. scient. Éc. Norm. Sup., $4^{e}$ série, t. 14, (1981), 249-260.

[FIK] M. Feldman, T. Ilmanen, and D. Knopf, Rotationally Symmetric Shrinking and Expanding Gradient Kähler-Ricci Solitons, J. Diff. Geom., 65, (2003), 169-209. 
[G] Z. D. Guan, Quasi-Einstein Metrics, Int. J. Math., 6, (1995), 371-379.

[GK] A. Gastel and M. Kronz, A family of Expanding Ricci Solitons, Variational problems in Riemannian geometry, Prog. Nonlinear Differential Equations Appl. 59, Birkhäuser, Basel (2004), 81-93.

[GT] D. Gilbarg and N. S. Trudinger, Elliptic Partial Differential Equations of Second Order, Second Edition, Springer-Verlag, (1998).

[Ha1] R. S. Hamilton, The Ricci Flow on Surfaces, in Mathematics and General Relativity (Santa Cruz, CA, 1986), Contemp Math., 71, Amer. Math. Soc., (1988), 237-262.

[Ha2] R. S. Hamilton, Eternal Solutions to the Ricci Flow, Jour. Diff. Geom., 38 (1993), 1-11.

[Iv] T. Ivey, New Examples of Complete Ricci Solitons, Proc. AMS, 122, (1994), 241-245.

[Ko] N. Koiso, On Rotationally Symmetric Hamilton's Equation for Kähler-Einstein Metrics, Adv. Studies Pure Math., 18-I, Academic Press, (1990), 327-337.

[La] J. Lauret, Ricci Soliton Homogeneous Nilmanifolds, Math. Ann. 319, (2001), 715-733.

[PTV] H. Pedersen, C. Tønnesen-Friedman, and G. Valent, Quasi-Einstein Kähler Metrics, Lett. Math. Phys., 50, (2000), 229-241.

[Per] G. Perelman, The Entropy Formula for the Ricci Flow and its Geometric Applications, arXiv:math.DG/0211159.

[Pk] L. Perko, Differential Equations and Dynamical Systems, Springer Texts in Applied Mathematics, 7, Springer-Verlag, (1996).

[PS] F. Podesta and A. Spiro, Kähler-Ricci Solitons on Homogeneous Toric Bundles I and II, arXiv:math.DG/0604070, 0604071.

Jesus College,, Oxford University, OX1 3DW, United Kingdom

E-mail address: dancer@maths.ox.ac.uk

Department of Mathematics and Statistics, McMaster University,, Hamilton, Ontario, L8S 4K1, CANADA

E-mail address: wang@mcmaster.ca 